\title{
Charting the Research Course for Sustainable Aquaculture in Sabah, Malaysia
}

\author{
L. W. Vun, C. M. Payus and S. A. Mohd Ali \\ Faculty of Science \& Natural Resources, Universiti Malaysia Sabah, 88400 Kota Kinabalu, Sabah, Malaysia
}

\begin{abstract}
Due to arising needs and demands, aquaculture is currently the fastest growing food production sector. In order to increase yield and yet to remain sustainable, the challenges would be to minimise impact on the environment and ecosystem services. Aquaculture activity contributes significantly to Malaysia and also the state of Sabah's economy and food security. Hence, the future changes in the environment as a result of rapid population growth and development would pose as threats to this industry in terms of quality, quantity and sustainability. Unforeseen environmental changes such as environmental pollution from other sources, climate change and the changes in policies would jeopardize the sustainability of this industry. In order to anticipate such impacts to the aquaculture activities, this paper set to chart a sustainable course for its development. Four important research courses were proposed: establishment of a sustainable framework, assessment of impacts of climate change, viability and vulnerability assessment due to future environmental changes and food security. Such findings would eventually allow the stakeholders to plan and manage the resources and aquaculture activities in such a way that foster sustainable food security and resilient aquatic ecosystems.
\end{abstract}

\section{Introduction}

Aquaculture is currently the fastest growing food production sector and it is expected to supply over half of the world's seafood [1]. With the arising needs and demands, Wilfart et al. [2] foresees that aquaculture will face four inevitable challenges: increasing cultivable areas without decreasing biodiversity or increasing water demand, improving food quality, producing ecosystem services and adapting to climate change. Thus, aquaculture faces the pressure to increase yield yet be sustainable.

In Sabah, aquaculture has great potential to contribute to the state's economy and food security, although it is not as significant as the palm oil industry, it is promoted as a promising commercial venture to meet consumer demand for seafood and support community livelihoods in general [3]. It is inevitable that there will be changes to the structure of the activity (from small scales to corporate), the coverage (from meeting domestic needs to meeting regional and global needs), the intensity, the approach (from conventional to innovative) and also the stakeholders (from individual to local to national to multiplayers).

Hence, aquaculture is vulnerable to the impact of climate change, future variability and changes in the environment as a result of rapid population growth. This would mean that aquaculture production in the state will be exposed to the threats of future environmental changes to its quality, quantity and sustainability.
Continued growth in aquaculture production is likely to set off intensification of production and this would bring about a range of resource and environmental problems. Yet at the same time, this sector is at risk due to possible future environmental changes, particularly as aftereffects of climate change and increased anthropogenic wrong doings. Climate change is only one among many environmental and anthropogenic stresses faced by aquaculture but is likely to complicate the process of achieving sustainability [4].

\section{The need for a sustainable course}

The first question that we pose in this scenario is how to ensure that this growing sector will incorporate sustainable practices and avoid some of the possible resource and environmental problems that have plagued the agricultural and livestock sectors in the past decades. The second question we ask is how the aquaculture sector can anticipate the impacts of future environmental change.

The answer to the first question have been aptly addressed by Klinger and Naylor [5] in a recent review, they have managed to identify some of the most promising pathways toward sustainable growth in the future of aquaculture sector. They raised three main issues: the technological innovations related to energy efficiency, the waste management matter and the genetic technologies for the enhancement of terrestrial aquafeed ingredients. They acknowledged that most innovative, productive, profitable, and environmentally sound 
systems are likely to vary by species, country, and policies.

In view of this, we attempted to propose measures to address the second question on charting a course for Sabah, so that the future of development in aquaculture in the state will be on a sustainable course.

Anticipating future environmental changes such as climate change and anthropogenic stresses will enable policy-makers and the stakeholders to be more aware and alert of the future challenges, and they could plan and manage the resources and activities in such a way that foster sustainable and resilient aquatic ecosystems. As this will benefit the aquaculture industries, and also provide goods and services at the national and even global levels, for example, through sustained food security and the conservation of biodiversity.

\section{Charting a sustainable course}

When attempting to meet sustainability, I firmly believe that sustainable development comes after taking into due account the future sustainable opportunities for the future generations. The simple idea would be as follows:

Resources - Future Sustainability and Opportunities $=$ Sustainable Development

As clearly started by Serageldin [6] and in his own words: sustainability requires leaving to the next generation exactly the same amount and composition of natural capital as we found ourselves, and to substitute a more promising concept of giving future generations the same, if not more, opportunities than we found ourselves.

Thus, we attempt to address the future aspect first by developing a sustainable framework for aquaculture activities, and then follow by preparing the industry in anticipating future environmental changes.

Smil [7] in his book on Global Catastrophe and Trends: The Next Fifty Years remarked that any prediction of the future environmental changes could potentially be hampered by two incessant processes: the ever changing existing trends and also the shifting significance and concerns due to interactions of current and underestimated trends. However, Smil also mentioned that catastrophes and endings are also opportunities and beginnings. Taking these into consideration, we believe that a framework would serve as a basis for us to begin with (in moving towards sustainability) and also a platform for us to deal with any imminent negative environmental changes.

\subsection{Sustainable framework for aquaculture}

Developing a sustainable framework for aquaculture activity in Sabah will provide valuable understanding of the limit and provide the standards for the industry. The identified and developed suits of indicators will enable all stakeholders to know the rhythm of the aquaculture activities, symptoms and signs are good indicators that foretell possible problems and concerns.

Sustainability indicators also enables monitoring and reporting on the state of the aquaculture activities and relevant ecosystems locally and regionally. By taking into account a broad spectrum of sustainability perspectives and concerns, a comprehensive framework for the assessment can therefore be proposed, and results communicated to the decision making process. Although this does not necessarily offer a definitive judgement on sustainability, but present a holistic view, allowing recognition of trade-offs or compromises involved between conflicting sustainability objectives if any.

\subsection{Impact of climate change on aquaculture}

Understanding the impact of climate change on aquaculture is necessary on the research agenda as climate change is inevitable. Review of local and regional adaption and mitigation strategies related to aquaculture sector in view of climate change will allow stakeholders to anticipate such impacts [8].

This issue is also highly relevant to the formation of Malaysia's national climate policies and strategies. With the close of the recent COP21 (2015 Paris Climate Conference), the commitment to tackle climate change will again be renewed and new targets will be pledged. Thus, it is important to identify the impact of this on aquaculture as it posed as one of the most vulnerable economic sector, especially in developing countries like Malaysia. Of course the actual impact will be hard to predict, but due consideration can be given to the different impacts of climate change over a period of time, whether they are positive or negative.

\subsection{Effects of environmental changes on aquaculture}

Open-intensive aquaculture activities would have an impact on the water environment, nonetheless, this activity is susceptible to the changing environment, and such as the decline in water quality will affect the long term sustainability of the aquaculture activity. Thus, it is important to carry out long term and periodical monitoring to look at the potential sources of environmental pollutants that might threaten this activity, so that the management of this issues could be aptly addressed. Understand the risks of aquaculture activities in relations to nutrients salts, medication and also organic pollutants within the vicinity. This would enable the establishment of standards for continuous monitoring and food safety.

Once we understand the pollutants in the environmental, we would be able to implement useful measures, such as the 4S strategy (Stop, Slow, Simplify and Share) in dealing with environmental problems [9].

\subsection{Aquaculture and food security}

No matter how much we consider the issue of sustainability, ultimately it is the food security for the present and future generations that must be addressed.

The impact of aquaculture on the environment and local communities: issues on food security, food nexus, access to and management of common pool resources, could result in conflicts with exiting users and potentially 
acute social, political, and economic problems [10]. So, partnership is a must for this to be addressed.

\subsection{Aquaculture and ecosystem health}

Ecosystem health is closely related to aquaculture and this depends greatly on how the farmers, aquabusiness, aquaculture scientists, local communities and policy makers to understand the interrelationships and work towards tapping into what aquaculture activities can offer towards ecosystem health. Ecosystem health has been a neglected aspect for most development as the approach tends to drain certain services and sometimes even cause disservice. Aquaculture activities could well be a double edged sword as it could also provide beneficial environmental services if governed and practised in an ecosystem approach and an integrated manner. However, such ideal or sustainable use of goods and services and impacts on ecosystem integrity depend greatly on our understanding of the system dynamic. Further research is required to answer this because to achieve such a balance is very challenging.

\subsection{The sustainable aquaculture framework}

Thus, to achieve sustainability in this activity, having a sustainable framework would be a good start. As this would provide the minimal framework for all the stakeholders to begin their work with. Furthermore, an existing framework would serve as a precautionary step to move forward. Oversight in development would jeopardise the industry. And finally, a framework would help the stakeholders to take action on any future environmental changes that might have impacts on the industry.
Figure 1 depicts the framework proposed in this paper towards sustainable aquaculture. The box in the middle shows that farming activities and processing have an effect on the water quality, that is why technical options are important as they play a role in identifying the most effective and efficient strategy to reduce such impact. Dotted boxes show the important factors and players that will influence aquaculture activities, minimal environmental changes and healthy ecosystem services would provide the desired environment for the industry to thrive in.

Environmental policies changes (e.g. climate change policy) will also define the direction of the industry. The same goes for governance and best management practices as they would also shape the aquaculture activities. One last but not least is the partnership from the local communities. Taking all these into account would make a good alliance in bringing the industry towards sustainable aquaculture.

The viability of a "sustainable aquaculture" industry would depend on a collaborative and malleable process that allow all the stakeholders to adapt and communicate in order to establish, shift and deploy any policy goals [11].

From the business front, there is a need to experiment with new ways of conducting the business affairs, according to Williams [12], businesses that fail to take responsibility, embrace transparency and open up to new collaboration will increasingly seem out of touch in this green era. Thus, Williams has proposed a New Behavioural Contract that allows the businesses to try-out innovative and never-thought-possible ways of working together towards sustainability. A new sustainable paradigm shift for the businesses.

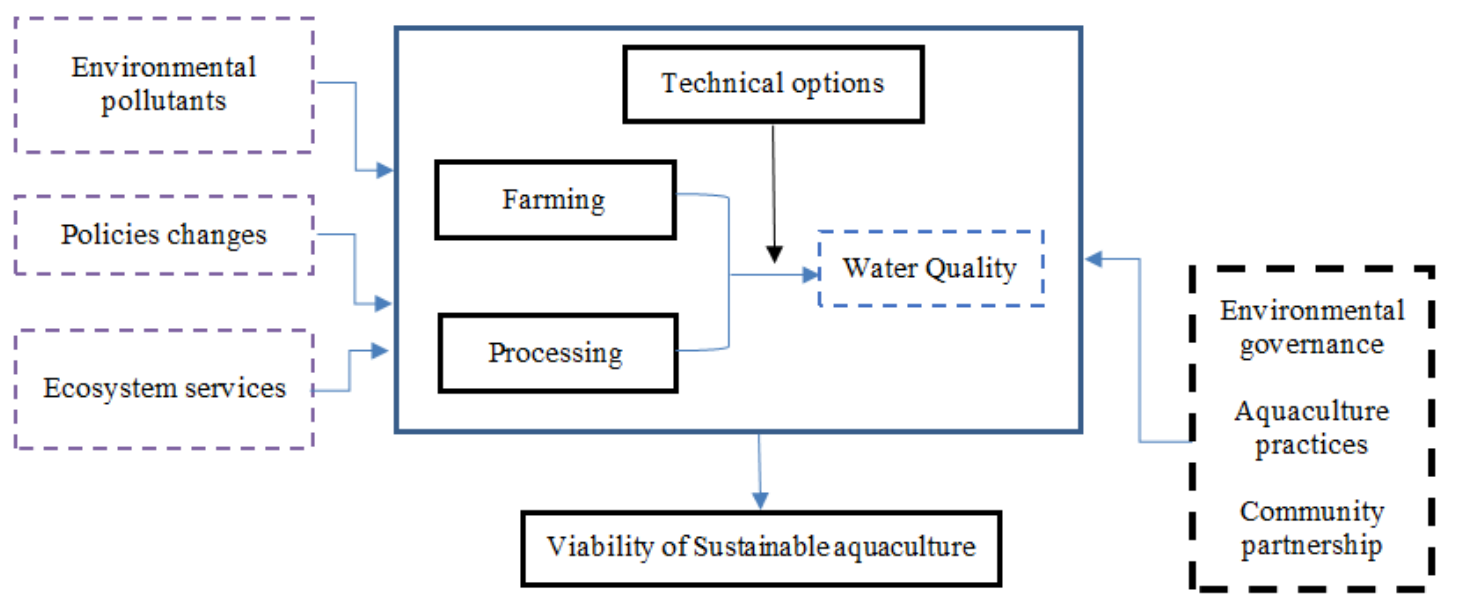

Figure 1. Framework towards sustainable aquaculture for Malaysia.

\section{Conclusion and suggestion}

By anticipating future environmental changes such as climate change and anthropogenic stresses will empower policy-makers and all stakeholders to be more alert of the future challenges, and they could plan and manage the resources and activities in such a way that foster sustainable and resilient aquatic ecosystems. This will not only benefit the aquaculture industries, but it will also provide goods and services at the national and even global levels, for example, through sustained food security and the conservation of biodiversity.

The proposed framework for sustainable aquaculture will help to organize and guide the planning to move forward while leveraging and incorporating all stakeholders' input as well. 
With the presence of a framework, the organization would help to put everything into proper perspectives, remove any potential assumptions and sort out what is the best step(s) to go forward based on the best available knowledge and information about the industry and also the environmental dynamics.

The framework will also incorporate others' thinking and ideas. In this process, not only that the precautious principles are applied, it also could be an eye opener to realise that there are better ideas that could solve potential problems, such as local knowledge that could address the same issue without carrying out any time and cost consuming researches for that matter. With such inputs, they will also stimulate the thinking in others to propose ideas that are even better. Thus, this framework can also be viewed as a platform or starting point for the stakeholders to interact with one another and decide on the best solution.

Critical gaps in planning and application, policies and enforcement could be identified and filled, hence the implementation of adaptation and mitigation measures would be possible. Raised awareness and well managed aquaculture activities play important roles also in maintaining healthy and productive ecosystems and vice versa. Indeed, it is the economic returns that will drive and influence and farmers' production decision. However, through awareness, farmers could learn that sound environmental responsibilities actually make good business and create wealth and that pollution will lead to the outbreak of diseases and this is not business smart.

The two main purposes of sustainable aquaculture are for the sustained business for the farmers and also for the sustained and desirable healthy generations to come because the food source is meant for all but it is not just about feeding but staying healthy. Thus, the research course helps to move the industry towards that end.

In principle, the $4 \mathrm{~S}$ approach would help: Stop, Slow, Simplify and Share [9]. Collectively, if all the stakeholders share the same approach, this would help to regulate the course for sustainable aquaculture to be always on the right track. For example, stakeholders united to stop the use of drugs, slow down the intensification that might cause the misuse of unhealthy seeds, simplify certain processes and share or advocate water recycling or treatment before discharge would promote a shared partnership towards environmental protection. Only in protecting the viability, vitality and diversity of the environment that we can ensure sustainability.

In propagating industrial and agricultural activities in the past, mistakes have been made because of ignorance, carelessness and the lack of restrain. As one moves towards a surging and challenging industry, we should embrace prudence and seek to create a desirable environment for us and also the future generations.

Charting the research course for sustainable aquaculture in Sabah, Malaysia is to be ahead of the game. We especially need committed proactive and aggressive management of resources for sustainability.

\section{Acknowledgement}

The authors thank the Ministry of Higher Education (MOHE), Malaysia, in providing the Niche Research Grant Scheme (NRGS) for this project. The authors also thank Isaac W. Witus, Weipeng Lee, Vincent Woon, Peirong Han, Nurul Fazlina for being a part of this project.

\section{References}

1. D. Cressey Nature, 458, 398-400 (2009)

2. A. Wilfart, J. Prudhomme, J. P. Blancheton, J. Aubin, Journal of Environmental Management, 121, 96-109 (2011)

3. SEDIA. http://www.sedia.com.my. (Accessed on 06.01.2016)

4. FAO, Climate Change For Fisheries And Aquaculture. Technical Background Document, Rome: FAO (2008)

5. D. Klinger, R. Naylor, Annu. Rev. Environ. Resourc., 37, 247-276 (2012)

6. I. Serageldin, Making Development Sustainable: From Concepts to Action, World Bank (1994)

7. V. Smil, Global Catastrophe and Trends: The Next Fifty Years, MIT Press (2012)

8. FAO, Climate change implications for fisheries and aquaculture, FAO Rome (2009)

9. L. W. Vun, International Journal of Environmental Studies, 72, 599-600 (2015)

10. B. A. Costa-Pierce, D. M. Bartley, M. Hasan, F. Yusoff, S. J. Kaushik, K. Rana, D. Lemos, P. Bueno, A. Yakupitiyage, Responsible use of resources for sustainable aquaculture, Global Conference on Aquaculture 2010, Sept. 22-25, 2010, Phuket, Thailand, Food and Agriculture Organization of the United Nations (FAO), Rome, Italy (2011)

11. E. A. Wolters, B. S. Steel, D. Lach, D. Kloepfer. Ocean \& Coastal Management, 122, 95-102 (2016)

12. E. F. Williams, Green Giants: How Smart Companies Turn Sustainability Into Billion-dollar Businesses, Amacom (2015) 